ArtefaCToS. Revista de estudios de la ciencia y la tecnología

eISSN: $1989-3612$

Vol. 8, No. 2 (2019), 2. ${ }^{a}$ Época, 103-124

DOI: http://dx.doi.org/10.14201/art201982103124

\title{
Normatividad y deliberación práctica en John Dewey: elementos para una lectura contemporánea
}

\section{Normativity and practical deliberation in John Dewey: elements for a contemporary reading}

\author{
Federico Ezequiel LÓPEZ; \\ ${ }^{*}$ Universidad Nacional de La Plata, Argentina \\ flopez@fahce.unlp.edu.ar \\ **Universidad Nacional de La Plata / \\ Consejo Nacional de Investigaciones Científicas y Técnicas, Argentina \\ livio.mattarollo@nacio.unlp.edu.ar
}

Recibido: 05/07/2019. Revisado: 08/07/2019. Aceptado: 16/08/2019

\section{Resumen}

El propósito de este trabajo es explorar la respuesta de John Dewey a la disputa que durante siglos había enfrentado a escépticos y dogmáticos acerca de la cuestión de la normatividad. La estrategia principal de Dewey consiste en elaborar una hipótesis para explicar cómo los principios normativos o de jure tienen un anclaje y un punto de partida en la actividad práctica, de facto. A efectos de reconstruir la concepción de la normatividad de Dewey, y teniendo en cuenta las malas interpretaciones de las que ha sido objeto, en primer lugar, analizaremos la naturaleza del problema -problema que, desde el punto de vista de Dewey, es político. En segundo lugar, mostraremos de qué modo la concepción de la racionalidad de nuestro autor resulta divergente respecto de la concepción dominante que, apelando a la todavía vigente distinción entre contexto de descubrimiento y de justificación, hace de la justificación su componente básico. En tercer lugar, y luego de destacar la centralidad que tiene en el pensamiento de Dewey el proceso práctico de resolución de problemas como núcleo de la racionalidad, analizaremos su concepción de la deliberación práctica que se constituye de este modo como el origen de toda normatividad.

Palabras clave: escepticismo; dogmatismo; racionalidad; razonamiento práctico; principios morales. 


\begin{abstract}
The aim of this paper is to explore John Dewey's solution of the problem of normativity that has confronted skeptics and dogmatists for centuries. Dewey's main strategy is to elaborate a hypothesis to explain how the de jure or normative principles have an anchor and a starting point in practical, de facto activity. In order to reconstruct Dewey's conception of normativity, and taking into account the misinterpretations it has received, we will first analyze the nature of the problem -a problem that, from Dewey's point of view, is a political one. Second, we will show how our author's conception of rationality diverges from the dominant conception, which making reference to the still prevalent distinction between the context of discovery and the context of justification, takes justification as its basic component. Third, after emphasizing the centrality of the practical problem-solving process as the core of rationality in Dewey's thought, we will analyze his conception of practical deliberation, which is thus seen as the origin of all normativity.
\end{abstract}

Keywords: skepticism; dogmatism; rationality; practical reasoning; moral principles.

\title{
1. Introducción
}

El problema de la normatividad, esto es, el problema de explicar qué es una norma, cómo funciona y de dónde deriva su carácter racionalmente vinculante, ha sido, aunque bajo formulaciones diferentes, un objeto de reflexión fundamental a lo largo de la historia de la filosofía. El intento de definir, aclarar y expandir los conceptos mediante los cuales se intentó dar respuesta a tales interrogantes ha motivado, una y otra vez, el surgimiento de posiciones tanto escépticas como dogmáticas. Las discusiones antiguas en torno al criterio de verdad, el intento cartesiano de convertir la bondad de Dios en fundamento de los criterios de claridad y distinción, e incluso las iniciativas más contemporáneas de fundamentar el principio de inducción pueden considerarse como episodios de tal debate. En efecto, la pretensión de saldar dicha discusión, de un modo u otro, ha motivado a muchos filósofos y en ello el pragmatista John Dewey no constituye una excepción.

Pese a que, en su opinión, escépticos y dogmáticos son víctimas de un error metodológico compartido consistente básicamente en el olvido del contexto, el problema que abordamos no era para Dewey un mero problema filosófico sino más bien un problema vital, o mejor aún, era un problema filosófico porque era un problema vital. Puede decirse, como señala Vincent Colapietro (2018), que el problema se origina y se reactualiza en las crisis de autoridad que mujeres y hombres han experimentado a lo largo de la historia y que se trata, por tanto, 
de un problema eminentemente práctico: la acción humana se encuentra regida por normas o principios, de modo que la pregunta por cuáles de ellos merecen nuestra aceptación se vuelve un problema crucial. Frente a tal cuestión, Dewey ha elaborado una respuesta profunda e interesante, que, sin embargo, no ha sido suficientemente tenida en cuenta o explorada por muchas tradiciones filosóficas que aún hoy intentan encontrar una solución al problema. En efecto, la recepción sesgada del pragmatismo en general y de las ideas Dewey en particular forjó la idea de que este último fue un pensador que, desde un punto de vista filosófico, tuvo algunas ideas muy interesantes pero que, desde un punto de vista lógico $o$ argumentativo, las defendió de un modo poco riguroso y un tanto confuso, cuando no completamente defectuoso. En este marco, por ejemplo, la concepción deweyana de la ética y de la valoración han sido objeto de fuertes críticas (Lewis, 1929; Marcuse, 1941; Stevenson, 1944; White, 1949), lo que abonó un sentido común filosófico según el cual el pragmatismo, y Dewey en particular, no tienen una solución adecuada al problema de la ética, esto es, al problema de la fundamentación de los principios o normas morales ${ }^{1}$.

Frente a ello, este trabajo pretende llamar la atención sobre algunos aspectos valiosos de la "solución" deweyana que, creemos, merecen ser analizados con mayor detalle. A efectos de comprender tal solución resulta importante tener presente la estrategia adoptada por Dewey, consistente en analizar la cuestión de un modo naturalista y contextualizado: si la inteligencia no es más que una capacidad desarrollada por el ser humano como producto de la evolución, entonces las normas -como elementos propios de las actividades que llamamos inteligentes- deberán comprenderse como emergentes de ese proceso. Así, antes de responder la pregunta acerca del por qué del carácter vinculante de las normas, Dewey se pregunta por su origen, que no es, en su opinión, otro que la actividad de resolución razonada de problemas, es decir, de la actividad mediante la cual decidimos qué hacer para superar una cierta dificultad. En otros términos, Dewey encuentra en la deliberación práctica el origen de toda normatividad y es apelando a la posibilidad de encontrar respuestas suficientemente adecuadas a problemas prácticos como pretende explicar de un modo a la vez anti-escéptico y anti-dogmático qué es una norma, cómo funciona y de dónde obtiene su carácter racionalmente vinculante. Al hacer eso, Dewey propone una concepción de la razón misma que ha resultado muchas veces, por su literalidad radical, difícil de valorar en sus profundas consecuencias.

A efectos de analizar esta posición, y teniendo en vista su posible reapropiación contemporánea, el recorrido que proponemos en este trabajo es el siguiente.

\footnotetext{
${ }^{1}$ En términos generales, los interlocutores de Dewey al momento de reflexionar sobre valoración son dos: el enfoque no-naturalista y cognitivista del apriorismo, por un lado, y ya en la década de 1930, el enfoque naturalista y no cognitivista del emotivismo ético, por el otro, en vistas a ofrecer una teoría de la valoración naturalista y cognitivista (cf. Di Berardino y Faerna, 2008, 13). Respecto de la discusión entre Dewey y el emotivismo ético, cf. Faerna (2006, 2011).
} 
En primer lugar, exploraremos la naturaleza del problema. En tal exploración sacaremos a la luz la dimensión eminentemente moral y política del mismo, que se corresponde con la dimensión igualmente moral y política de la solución propuesta por el pragmatista. En segundo lugar, explicitaremos algunos supuestos que, en nuestra opinión, están a la base de cierto extrańamiento con el que los lectores contemporáneos a Dewey e incluso los actuales suelen recibir sus ideas. En efecto, la posición del pragmatista supone un modo radicalmente divergente de comprender la racionalidad y tal radicalidad debe ser tenida en cuenta a la hora de estudiar su pensamiento. En este sentido sostendremos que, frente al primado contemporáneo de la relación razón-conclusión como componente básico de la idea de racionalidad, la concepción de Dewey asume de un modo consecuente el carácter fundamental, o si se quiere originario, de la relación problema-solución, adquiriendo así una impronta práctica. Luego, analizaremos la concepción deweyana de la deliberación práctica que, en tanto que estructura de la resolución razonada de problemas, se constituye en la pauta de la racionalidad y en la fuente de toda normatividad. Para concluir, destacaremos algunos aspectos de la posición de Dewey que, creemos, deberían ser considerados por los intentos contemporáneos de reconstruir la lógica del razonamiento práctico.

\section{Escepticismo, dogmatismo y la salida hacia la práctica}

La disputa entre dogmatismo y escepticismo es un asunto que ha ocupado a los filósofos desde el comienzo mismo de su disciplina. Desde entonces, los participantes de tal disputa han tratado de refutar el punto de vista de sus contrincantes, representando el rol opuesto. A finales del siglo XIX y aún más durante el siglo XX, apareció un nuevo personaje que, en vez de optar por uno de esos roles, trató de superar el conflicto argumentando que escepticismo y dogmatismo son dos caras de una misma moneda. Esta es precisamente la estrategia del pragmatismo estadounidense clásico, que intentó construir una concepción del conocimiento y de la racionalidad anti-escéptica y anti-fundacionalista. La larga duración de esta controversia no se debe, al menos no exclusivamente, a la afición de los filósofos por los problemas sin solución, sino más bien a la naturaleza misma del problema. Como señala Colapietro,

[L]a derrota de la certeza del sentido común y, a continuación, el vuelco a un escepticismo en sí mismo aparentemente invencible, son episodios inherentemente dramáticos en la historia real de la reflexión filosófica. Pero son algo más que partes integrantes de un drama intelectual jugado en los enclaves de los intelectuales profesionales. Son dramas verdaderamente culturales de gran alcance, internalizados en actores que tienen poca o ninguna comprensión de los cambios de época y de las crisis definitorias del momento. $(2018,10)$.

Siguiendo las consideraciones sociológicas de Dewey respecto de la filosofía, Colapietro sugiere que tal drama cultural tiene un carácter eminentemente políti- 
co. Tomando algunas interesantes observaciones que Dewey presentó en un texto temprano titulado "The Significance of the Problem of Knowledge" (ew.5.3-24), Colapietro señala que las crisis de autoridad epistémica están estrechamente vinculadas con crisis de autoridad política $(2018,10)$. El carácter doble, epistemológico y político, de la querella que enfrenta a dogmáticos o fundacionistas -como se les suele llamar en la actualidad-y escépticos es probablemente la razón por la cual en el contexto de la filosofía contemporánea el concepto de racionalidad ha estado en el centro de la controversia. De hecho, este concepto permite tratar a la discusión epistemológica, política y ética de una manera unificada.

Esta relación interna y sistemática entre cuestiones epistemológicas y políticas es crucial en el pensamiento de John Dewey y es la razón por la que, a lo largo de toda su vida intelectual, estuvo tan preocupado por cuestiones de lógica. En su opinión, una comprensión adecuada de la lógica permitiría superar los obstáculos que impiden la aplicación del método científico o experimental, vale decir, del método de investigación reflexiva, a las cuestiones éticas y políticas. Para Dewey, ello no sólo es posible y legítimo, sino además urgente, una necesidad de su tiempo. En los términos de Colapietro, el drama al que se enfrentaba Dewey era que la inteligencia no se empleaba para enriquecer y ampliar la experiencia humana, para analizar las costumbres y los valores establecidos ni para formar otros nuevos. Según Dewey, esta falencia resulta particularmente relevante al momento de considerar los fines y valores que guían la producción de conocimiento científico, pues, en su opinión,

La ciencia es un instrumento, un método, un cuerpo de técnicas. Si bien es un fin para aquellos investigadores que se dedican a su prosecución, en el sentido humano amplio es un medio, una herramienta. ¿Para qué fines habremos de utilizarla? ¿Será utilizada de forma deliberada, sistemática, para la promoción del bienestar social, o será empleada principalmente para el engrandecimiento privado, dejando sus resultados sociales más amplios librados a la casualidad? ¿Será que la actitud científica se puede utilizar para crear nuevas actitudes mentales y morales, o seguirá subordinada al servicio de deseos, propósitos e instituciones que se formaron antes de que la ciencia existiera? (lw.6.54-55) ${ }^{2}$.

\footnotetext{
${ }^{2}$ Las citas y referencias a Dewey se harán a las versiones en español, cuando las hubiera. En caso contrario, las traducciones de títulos y fragmentos serán propias. Como es habitual en la literatura especializada, en todos los casos se remitirá a las obras completas: The Collected Works of John Dewey, The Early Works, 1882-1898 (1967-1972); The Middle Works, 1899-1924 (1976-1983); The Later Works, 1925-1953 (1981-1991). Así, en caso de haber traducción al español la referencia quedará consignada como Dewey, año, página, junto con la cita de la obra completa, ew/mw/ lw.volumen.página. La única excepción es el libro Unmodern Philosophy and Modern Philosophy de Dewey (2012) cuya edición y publicación es posterior a la edición de los referidos Collected Works. Las citas y referencias a Peirce se harán a las Collected Papers (Peirce, 1965).
} 
Este párrafo invitar a considerar varias cuestiones. En primer lugar, y más allá de que a primera vista Dewey parece adoptar una visión de la ciencia como un instrumento neutral, en el sentido de que pudiera ser empleada con diferentes fines, su tesis de la continuidad entre medios y fines es tal que los medios específicos que la inteligencia crea contienen ya el fin a la vista (cfr. lw.13.214 y ss.), de modo que la consideración de la ciencia como medio no puede desligarse de la consideración sobre sus fines. En otros términos, cada desarrollo científico en particular contiene la marca, por así decirlo, del fin con el que fue pergeñado, aun cuando la ciencia, en sentido general, pueda emplearse con diferentes fines. En efecto, el pasaje es más bien una invitación a reflexionar profundamente sobre la dimensión política de la ciencia. En sus palabras, "[n]os vemos obligados a considerar la relación de las ideas e ideales humanos con las consecuencias sociales que se producen por la ciencia como un instrumento" (lw.6.55). Esta reflexión urgente tenía, y probablemente tiene hoy más que entonces, un carácter dramático e incluso trágico. En este sentido, Dewey plantea que "[e]l problema en cuestión es el más grande al que la civilización ha tenido que hacer frente. Es, sin exagerar, el problema más grave de la vida contemporánea" (lw.6.55). El tono dramático de este pasaje está presente también en la interpelación que agrega a continuación: "He aquí el instrumento, el más poderoso, para bien y para mal, que el mundo ha conocido. ¿Qué vamos a hacer con él?” (lw.6.55). Frente a este problema, entonces, Dewey se pregunta acerca de los principios que hemos de reconocer como legítimas guías en nuestras deliberaciones, por ejemplo en aquellas referentes a los fines de la ciencia. En relación con ello, una respuesta escéptica sugeriría que no hay forma racional de decidir sobre esta cuestión, mientras que una respuesta dogmática sugeriría que no hay nada que decir, pues los principios, las normas básicas, están ya fijados de una vez y para siempre, sea por Dios o por la razón. Por su parte, Dewey propone que sea la experiencia inteligente, la actividad humana reflexiva y científicamente informada, la que permita arribar a una decisión fundada sobre la cuestión, evitando a la vez postular principios racionales fijos y consagrar el deseo y el impulso, tal como emergen de hecho, como la única guía.

Sin embargo, en vez de facilitar esta tarea, la filosofía parecía impedirla o retrasarla debido a ciertas concepciones erróneas acerca de la inteligencia, el conocimiento y los valores, o en pocas palabras, sobre la racionalidad. Aunque no podemos explicar de manera detallada por qué, en opinión de Dewey, la filosofía ha desempeńado ese papel, incluso en contra de sus propios propósitos declarados, resulta importante señalar que, en su opinión, la filosofía moderna había conservado la idea antigua de que el conocimiento es una forma de contemplación de una realidad, que debe ser de naturaleza previa y fija para que sea pasible de aprehensión. La idea de Dewey es que esto era coherente con la práctica epistémica griega e incluso con la forma en que la sociedad griega estaba políticamente organizada (cfr. Dewey, 2012). Sin embargo, a partir de la revolución científica del siglo XVI, la ciencia misma [episteme] se volvió experimental; al hacerlo, descartó 
la dimensión cualitativa de la experiencia y se centró en los rasgos cuantitativos de la naturaleza, es decir, en el cambio. La consecuencia de todo esto es que, si el conocimiento es la contemplación de una realidad antecedente y fija y si la ciencia no considera cualidades y valores, entonces el conocimiento de los asuntos humanos, vale decir, de la ética y la política, deviene altamente problemático. En este sentido Dewey señala en La Búsqueda de la Certeza que la filosofía moderna se construye sobre una "división intestina" producto del colapso entre los referidos avances de la revolución científico-experimental y la estructura conceptual heredada de la filosofía griega sobre la naturaleza del conocimiento y de los valores (cf. Dewey, 1952, 47. lw.4.43).

Los filósofos modernos intentaron resolver este problema convirtiendo los valores en una especie de hecho que podría contemplarse y describirse (por ejemplo, un simple impulso o emoción) o mediante la elaboración de alguna forma de dualismo epistémico y ontológico que asegure un reino independiente, antecedente y fijo, en donde pudieran existir algo así como hechos racionales, susceptibles de ser contemplados. Sin embargo, ambas soluciones son problemáticas. La primera parece incapaz de explicar el hecho de que un valor o norma no es meramente una declaración de cómo son las cosas sino una estipulación de cómo deberían ser. ${ }^{3}$ En efecto, equiparar las normas con los impulsos, los deseos e incluso con las normas de hecho vigentes en un determinado momento, implicaría reducir toda normatividad a los valores, los deseos y las tradiciones que expresan los intereses de algún grupo en particular. El segundo introduce un dualismo ontológico o un sobrenaturalismo que, además de ser lógicamente problemático, está en desacuerdo con la idea bien establecida de que los seres humanos, y en consecuencia todas sus capacidades, son productos de evolución natural.

A efectos de superar estas posiciones, de acuerdo con Dewey, era necesario hacer a un lado "[l]a doctrina de la naturaleza estrictamente teórica o 'intelectual' del conocimiento" $(2012,252)$ y adoptar una concepción del conocimiento y la racionalidad práctica e incluso tecnológica, como sugiere en un interesante artículo titulado "By Nature and by Art" (lw.15). En su opinión, el pensamiento reflexivo y el conocimiento no es un intento de descripción de la realidad, sino más bien un tipo de práctica que involucra como una de sus etapas la formulación de juicios descriptivos o declarativos pero que no puede reducirse a ella. En su Lógica: Teoría de la Investigación Dewey afirma que

[...] la conducción de una investigación científica, ya sea física o matemática, es un modo de práctica. El científico es un práctico por encima de todo y se halla

\footnotetext{
${ }^{3}$ Como suele señalarse, Dewey rechaza cualquier tratamiento dicotómico de la distinción ser/ deber ser aunque era consciente, por ejemplo, de la diferencia entre decir que algo es deseado o deseable (Cf. Teoría de la Valoración, 2004. lw.13; Sinclair, 2014).
} 
constantemente embarcado en la emisión de juicios prácticos, es decir, en obtener decisiones acerca de lo que conviene hacer y de los medios a emplear para hacerlo. (Dewey, 1950, 183. lw.12.163) ${ }^{4}$

Así la actividad cognitiva, incluso en el ámbito científico, puede y debe explicarse como una actividad de toma de decisiones prácticas y el conocimiento no es más que un producto de esa actividad. Por supuesto que un científico deriva conclusiones, testea hipótesis y describe los resultados de sus observaciones, pero todas ellas son acciones que cobran sentido en el contexto de una actividad práctica. Más aún, son actividades intermedias que permiten producir los medios necesarios para la resolución del problema que enfrentan. En eso consiste la actividad de conocer, y sólo podemos calificar a algo propiamente como conocimiento en la medida en que es producto de la investigación. Desde la perspectiva de Dewey, cualquier intento de caracterizar el conocimiento y la racionalidad sin considerar el rol central de la investigación es erróneo, pues el conocimiento, al menos en un sentido epistemológicamente relevante, es literalmente el resultado de la investigación, aquello que obtenemos cuando hemos resuelto el problema que originó la investigación y por tanto ella misma ha cesado.

Por supuesto, hay situaciones en las que podemos decir que sabemos algo que no es el resultado de nuestra propia investigación. Pero, en esos casos, o bien es el resultado previamente establecido de alguna investigación, que no necesita ser reconsiderada, o es simplemente una pieza de tradición o sentido común, una información que se acepta sin demasiado análisis. Desde un punto de vista filosófico o lógico esta diferencia es crucial: en su sentido abstracto el concepto de conocimiento es, según Dewey, una generalización de las propiedades que se encuentran en las conclusiones particulares de un proceso que parte de una situación problemática y logra una solución satisfactoria. Si formamos un concepto de conocimiento mediante la generalización de las propiedades de las creencias que un sujeto tiene como resultado de la tradición o simplemente sin considerar cómo se alcanzaron, entonces se necesitará algún signo externo de verdad o de aceptabilidad para distinguir creencias "verdaderas" y creencias "falsas", un signo que deberá ser resultado de un proceso diferente de aquel a través del cual se han alcanzado las creencias. Por otro lado, si asumimos que "conocimiento" es nada más que el nombre de los productos de la investigación, entonces para explicar de manera más detallada por qué hay conocimiento "verdadero" y "falso" debemos estudiar el proceso de la investigación.

De este modo, la pregunta por la posibilidad del conocimiento, cuya respuesta oscilaba entre posiciones dogmáticas y escépticas, queda respondida por Dewey mediante la reconstrucción de lo que llama la lógica de la investigación y a partir

\footnotetext{
${ }^{4}$ El carácter práctico de la ciencia y su caracterización como un proceso de toma de decisiones y "selecciones" es recuperado por los estudios de laboratorio a finales de los ańos 70 (Bruno Latour y Steve Woolgar, 1995 [1986] y Karin Knorr-Cetina, 2005 [1981]).
} 
de la cual desarrollará su concepción práctica de la normatividad. Esto constituye el movimiento metodológico primario de la concepción del conocimiento de Dewey. Debido a la relevancia de este movimiento y a efectos de señalar algunas de sus consecuencias es importante considerar, al menos de forma esquemática, cómo la concepción de la normatividad de Dewey difiere de la noción básica o predominante de normatividad propia de la filosofía del siglo XX. En lo que sigue, pues, nos dedicaremos a analizar esta cuestión.

\section{Ni descubrimiento ni justificación: investigación}

Es un hecho bien conocido que la distinción entre contexto de descubrimiento y contexto de justificación ha jugado un papel importante en la filosofía contemporánea. Esta distinción, que recoge Hans Reichenbach (1938), junto con la proclamada superación del psicologismo (Kusch, 1995), son dos de los pilares sobre los cuales se construyó la filosofía del siglo XX, y en particular la tradición analítica. Como nos recuerda Larry Laudan, el establecimiento de esa diferencia supuso un cambio muy importante en el modo en que se desarrollaría la filosofía. En sus palabras:

Un acontecimiento de gran importancia ocurrió en el curso de la filosofía de la ciencia del siglo XIX. La tarea de articular una lógica del descubrimiento científico y la formación de conceptos, una tarea que había estado en el centro de la epistemología desde los Analíticos posteriores de Aristóteles, fue abandonada. En su lugar se colocó la tarea muy diferente de formular una lógica de la evaluación post hoc de teorías, una lógica que no se preocupa por cómo se generan los conceptos o cómo se formulan las teorías por primera vez. Esta transformación marca uno de los parteaguas centrales en la historia del pensamiento filosófico, una división fundamental entre dos perspectivas muy diferentes sobre cómo el conocimiento ha de ser legitimado." (Laudan, 1980, 175)

Pese a que esta distinción ha sido cuestionada en el campo de la filosofía de la ciencia al menos desde la década de 1960, lo cierto es que parece ser un dispositivo aún en funcionamiento. En opinión de Jutta Schickore y Friedrich Steinle (2006, ix), "el legado de los anteriores defensores de la distinción sigue siendo efectivo. La distinción continúa delineando el alcance de la filosofía de la ciencia” -y el de la lógica y la ética, nos permitimos agregar. Tanto es así que su supuesta confusión ha tenido el honor de recibir un nombre: la falacia genética (Cfr. Salmon, 1973). Más aún, quisiéramos sugerir que su influjo es una de las razones por las que el pensamiento de Dewey, y fundamentalmente su lógica, han tenido una recepción marcadamente crítica, llegando incluso a interpretarse como un sin sentido. En vistas de ello, resulta oportuno explicar de qué modo la concepción de la racionalidad de Dewey supone una tarea radicalmente diferente a aquella tarea de evaluación post hoc de teorías mencionada por Laudan. 
Como es sabido, la distinción entre contextos ha cumplido una función normalizadora al imponer a la filosofía de la ciencia, la lógica y la ética una tarea limitada: analizar teorías, redes conceptuales o simplemente creencias, es decir, la ciencia o la inteligencia como productos y no sus consecuencias ni su práctica misma, relegando el estudio de esto último a otras disciplinas o a áreas de la filosofía más bien secundarias. Dicho de otro modo, la posición más generalizada fue que para entender el conocimiento, tanto científico como moral, se podía y se debía prescindir de la consideración de los procesos mediante los cuales el conocimiento es de hecho producido. En este marco, el problema de la justificación se convierte en el asunto primario de la reflexión filosófica. Si, además, la justificación se comprende como un fenómeno absolutamente lingüístico, resulta que de la mano del giro lingüístico, al menos en sus dimensiones sintácticas y semánticas (cfr. Naishtat, 2006), la filosofía puede y debe limitarse al análisis de conceptos, teorías, marcos conceptuales, etc. y a sus propiedades lingüísticas.

Consecuentemente, la justificación se considera como una operación lingüística que se efectúa en virtud del soporte lógico que un conjunto de proposiciones (premisas) ofrece a otra proposición (conclusión). En otras palabras, la justificación es una cuestión de razonamiento, definido como un compuesto premisas-conclusión. Así, la relación premisas-conclusión se convierte en el núcleo mismo de la racionalidad: ser racional equivale a poder dar razón de nuestras creencias, y para lograr eso debemos ofrecer premisas, verdaderas o aceptables, a partir de las cuales cualquier ser racional extraiga, al menos idealmente, la misma conclusión.

Ahora bien, la idea de justificación entendida de este modo tiene un carácter fundamentalmente retrospectivo (cf. Toulmin, 2003, 6). El acto de justificar supone que el agente dispone ya de la creencia, la hipótesis o la teoría que pretende justificar. Su actividad racional consistirá en la búsqueda de fundamentos -o de objeciones y críticas en algunos contextos, como el popperiano. Resulta llamativo que se utilice la expresión "retrospectivo", en la medida que implica la idea de un proceso que va hacia atrás, invirtiendo de algún modo el orden que se da de hecho. Si el comienzo, en el sentido temporal, es la idea que se busca criticar o fundamentar, no se entiende por qué habría que calificarlo como retrospectivo. Sin embargo, como las ideas surgen de hecho en algún momento, parecería que sería posible analizar las condiciones lógicas de ese surgimiento. Las creencias que intentamos justificar, por ejemplo frente a otros, muchas veces son ocurrencias posteriores en un proceso que se ha iniciado antes. Sin embargo, ello nos conduciría a confundir descubrimiento y justificación. Frente a estas dificultades la respuesta ha sido negar toda connotación temporal a la idea de justificación, conservando sin embargo una idea de proceso retrospectivo que hace alusión a una prioridad lógica de las premisas por sobre la conclusión. Tal prioridad de las premisas se explica por el hecho de que el razonamiento tiene la función de preservar la verdad. Si hemos de justificar una creencia, tenemos que buscar premisas verdaderas, o al menos aceptables, cuya verdad o aceptabilidad se trasladará a la 
conclusión. Así, el núcleo mismo de la racionalidad se entiende como una operación retrospectiva en un sentido lógico, que busca fundamentos u objeciones para establecer el carácter de justificado o carente de justificación de una idea 5 .

En la medida en que estas ideas prevalecen e impregnan los modelos de solución de problemas -los paradigmas como ejemplares en el sentido de Kuhn- que se enseñan a las nuevas generaciones de filósofos, la concepción deweyana de la racionalidad resulta ciertamente incomprensible o, en el mejor de los casos, un esfuerzo filosófico bien intencionado pero mal orientado. Por ejemplo, la idea de que el conocimiento, la ciencia y el lenguaje general modifican y transforman sus objetos sólo puede interpretarse como postulando alguna fuerza mágica que crea o modifica la realidad en el acto intemporal de conocerla. Por otro lado, el fuerte carácter dicotómico de la distinción entre descubrimiento y justificación impidió que algunos intérpretes comprendieran que Dewey no trata de establecer una lógica del descubrimiento como diferente de una lógica de justificación sino que busca una lógica de la investigación como medio para lograr una aseveración garantizada. Este intento se basa en la idea de que la única razón vinculante para aceptar algo como conocimiento es precisamente ser el resultado de una investigación realizada correctamente. Cabe mencionar que, aun quienes han contribuido a cuestionar la distinción entre contextos, como es el caso de Toulmin, suelen conservar la idea de que la justificación de creencias constituye, de algún modo, el núcleo de la idea de racionalidad.

Sin embargo, ya desde finales del siglo XIX y principios del XX, Dewey había intentado desdibujar una distinción similar a la de descubrimiento y justificación, a saber aquella que hay entre génesis y validez. En "Valid Knowledge and the "Subjectivity of Experience" y discutiendo con aquellos que llama "nuevos realistas", Dewey reconstruye la objeción de su oponente de la siguiente manera: "Lo que objetamos, dirán, es la implicación de que las condiciones del descubrimiento de un objeto válido son las condiciones de su validez; nos oponemos a confundir las condiciones de la génesis con las condiciones de la validez" (mw.6.84). La respuesta de Dewey es lo suficientemente clara como para citarla in extenso:

La atención del realista es invitada a dirigirse a las conexiones sumamente íntimas y omnipresentes que existen entre las condiciones empíricas de génesis y consecuencia y el estatus de validez de un objeto. [...] La continuidad entre "experien-

\footnotetext{
${ }^{5}$ Otra consecuencia destacable de este centramiento descontextualizado en la idea de justificación radica en la reducción del lenguaje, desde el punto de vista del análisis lógico y epistemológico, a su función descriptiva y declarativa, en la medida en que el contexto de uso está por principio excluido de la consideración. Especialmente, como señala Dewey (cf. 1950, lw.12, cap IX), el uso del lenguaje mediante el cual pretendemos incidir y modificar una cierta situación, por ejemplo, en los pedidos pero también en la deliberación práctica, resultan completamente incomprensibles y carentes de toda propiedad lógica pues se piensan como oraciones que no constituyen verdaderas proposiciones.
} 
cia" y "validez" es a la vez tan inquebrantable y tan importante como para volver increíble la noción de que la génesis y las consecuencias estén en un ámbito -el subjetivo- y la validez en otro, el objetivo. (...) Aunque se pudiera considerar a la experiencia como subjetiva, no deberíamos pensar que hemos logrado nada con una distinción entre génesis y validez. [...] Deshacerse de la dificultad trazando una fuerte y tajante línea entre génesis y validez -o entre lo psicológico y lo lógico- es suponer que ponerle nombres a los términos del problema resuelve el problema que aquellos términos nombran. (mw.6.84)

Este rechazo a pensar en términos dicotómicos la relación entre génesis y validez se relaciona con la tesis antes mencionada según la cual la única razón que tenemos para llamar a algo conocimiento es que es el resultado de un proceso al que Dewey llama investigación o pensamiento reflexivo. Esta tesis supone tomar como relación básica, esto es, como unidad de análisis para la lógica y la epistemología, la relación entre un problema y su solución. A ello apunta la conocida definición de la investigación que Dewey propone en su Lógica: Teoría de la Investigación. La investigación es "[...] la transformación controlada o dirigida de una situación indeterminada en otra que es tan determinada en sus distinciones y relaciones constitutivas que convierte los elementos de la situación original en un todo unificado." (Dewey, 1950, 123. lw.12.108). En esta definición la investigación puede verse como un proceso en el que se llega a la creencia a partir de la duda, tal y como lo hace Peirce (CP.5.230). Pero en el pensamiento de Dewey se trata de algo más. Es un proceso en el que una seguridad o certeza relativa se construye a partir de un punto de partida incierto o indeterminado, construcción que supone mucho más que una manipulación de conceptos, ya sea deductiva o inductivamente. Tal transformación, operada por el organismo que conoce más que por una suerte de mente inmaterial, implica lo que Dewey denomina transacción, es decir, una forma de mutua modificación entre el organismo y el medio. Nuevamente, la relación básica que la lógica debe modelizar es la relación entre el problema y su solución, y no aquella que hay entre un conjunto de premisas y su conclusión.

Desde la perspectiva lógica de Dewey, no se trata de negar este componente inferencial, sino más bien de reubicarlo en su contexto para comprenderlo de modo adecuado. $\mathrm{Y}$ ese contexto no es meramente un proceso de descubrimiento, sino el proceso mismo de la investigación. Es decir, el problema no es cómo un investigador o una comunidad de investigadores arriba a una nueva idea sino cómo llega a una idea corroborada o garantizada, para usar la expresión de Dewey. No se trata simplemente de la justificación de una ida que ya tenemos, sino de la construcción de una solución que resulte adecuada. En el contexto de una investigación, no es un razonamiento lo que prueba la conclusión o solución, sino la investigación como un todo en la que el razonamiento es apenas una parte. Y esto vale tanto para conclusiones científicas, por así decirlo, como para conclusiones morales. Los principios morales a los que debemos dar nuestro consentimiento, aquellos que debemos permitir que guíen nuestra conducta, no son 
algo que debamos deducir de ninguna verdad previa ni intuir de modo directo, sino aquellos que nuestra investigación moral nos indique como los mejores. No se requiere de ningún elemento extraño o ajeno a ella.

Llegados a este punto, podemos preguntarnos -como lo hace Dewey- si es posible que esa forma de experiencia o de actividad humana que es la investigación o el pensamiento reflexivo pueda de hecho generar de modo autónomo sus propios principios regulativos, de forma tal que, desde el interior, permita explicar por qué algunas creencias deben considerarse como garantizadas y otras no. Dewey entiende que es posible comprender y explicar ese proceso en la medida en que reconocemos su carácter práctico, esto es, si reconocemos que toda investigación no es más que un proceso en el cual el agente decide qué acciones llevar a cabo. En otras palabras, se hace necesario reconocer que la pauta o la estructura de la investigación es la de la deliberación práctica.

\section{La lógica de la deliberación}

$\mathrm{Al}$ abordar la cuestión de la deliberación lo primero que es necesario recordar es que la deliberación práctica, es decir, la actividad de reflexión por medio de la cual consideramos cursos de acción y estándares de evaluación para decidir qué acción emprender, no es algo que se limite al campo de la técnica ni al de la ética. En efecto, de un modo inequívoco Dewey sostiene que "[...] el patrón característico de la deliberación se mantiene en el conocimiento científico" (2012, 266, nota, cursivas en el original). Más aún, puede sostenerse que la deliberación práctica es la estructura o patrón básico de la racionalidad o la inteligencia. ${ }^{6}$ Bajo estas consideraciones, si un problema no es meramente un estado mental sino un estado de la interacción organismo-medio y si su resolución implica transformar la situación problemática, entonces todo pensamiento reflexivo debería conducir, en caso de que sea completo, a la realización de una acción que transforme la situación como resultado de un proceso de deliberación. En otras palabras, y según hemos señalado anteriormente, toda investigación, en cualquier ámbito, es un modo de la práctica. Por supuesto, esto no implica que no haya diferencias entre una investigación científica, una de sentido común o incluso una deliberación ética. Lo que sí implica es que la estructura, la forma lógica, de todas ellas, no difiere en sus componentes metodológicos. Más aún, según indica Dewey en Lógica. Teoría de la Investigación, la pauta de la deliberación o de la investigación está ya anticipada en la actividad orgánica de transacción básica entre organismo y medio (1950, 37-56. lw.12.30-47).

\footnotetext{
${ }^{6}$ Aunque en algunas ocasiones Dewey rechaza los términos razón y racionalidad por su asociación con la tradición racionalista o intelectualista, como la llama en algunas oportunidades (cf. Volbers, 2018), y prefiere el uso del término inteligencia, lo cierto es que también intenta en ocasiones conferir un significado más bien pragmatista a tales términos (cf. p.e. Dewey, 1950, 22. lw.12.17).
} 
Ahora bien, volviendo a la pregunta con la que cerramos la sección anterior acerca de si la deliberación práctica puede operar de modo autónomo, la respuesta de Dewey es por demás sencilla, y encontramos en ella, además, el estricto paralelismo entre cuestiones científicas y valorativas antes mencionado. Para explicar dicha respuesta analizaremos algunos comentarios y argumentos que Dewey despliega en dos de sus obras más importantes, a saber su Lógica y su Teoría de la Valoración. En la primera de ellas, y refiriéndose a las investigaciones que contienen un factor práctico, el pragmatista sostiene que

[n]o son excepcionales ni raras investigaciones de este tipo ya que el acervo corriente del sentido común está compuesto de investigaciones y juicios de este género. Las deliberaciones de la vida conciernen en gran medida a cuestiones acerca de lo que conviene hacer. Todo oficio y toda profesión se enfrenta constantemente con problemas de esta índole. Dudar de su existencia equivale a decir que la práctica se halla desprovista de cualquier factor inteligente y que todas las decisiones de la práctica son producto arbitrario del impulso, del capricho, del hábito ciego o de la convención. El agricultor, el mecánico, el pintor, el músico, el escritor, el médico, el abogado, el comerciante, el industrial, el administrador, tienen que averiguar constantemente qué es lo que conviene hacer en el próximo momento. A menos que la decisión alcanzada se obtenga de forma ciega y arbitraria, se llega a ella reuniendo las pruebas por lo que respecta a su peso y significación; y fraguando planes de acción y probándolos en su capacidad de hipótesis, esto es, de ideas. (Dewey, 1950, 182-183. lw.12.162-163)

Luego de esta reflexión nuestro autor señala que no incluyó al científico en la lista de aquellos que investigan constantemente sobre qué hacer aunque inmediatamente después afirma que:

[...] basta pensar un poco para ver que también él tiene que decidir acerca de qué investigaciones iniciar y cómo llevarlas a cabo, problema que comprende, a su vez, el de las observaciones a realizar, los experimentos a practicar y las líneas de razonamiento y de cálculo matemático a seguir. Además, tampoco puede resolver estas cuestiones de una vez por todas. Continuamente tendrá que sopesar cuál sea el próximo paso conveniente para que su conclusión resulte fundada cuando llega a ella. (Dewey, 1950, 183. lw.12.163).

Nos interesa señalar algunos aspectos de ambas citas. En primer lugar, desde un punto de vista estrictamente lógico, Dewey sugiere que el razonamiento básico del científico es un razonamiento práctico, esto es, un razonamiento que concluye que alguna acción es la indicada, y que conduce así a la acción misma. Esta forma de comprender el razonamiento científico principal contrasta fuertemente con los modelos de "explicación científica", tanto con lo que se ha llamado el modelo nomológico-deductivo como con cualquier modelo estadístico o inductivo. Más aún, el razonamiento práctico, elemento fundamental de la actividad científica, no puede entenderse como un modelo ni hipotético deductivo ni inducti- 
vista de justificación de hipótesis. No se trata de que en la ciencia no se ofrezcan explicaciones o se apoyen hipótesis. Antes bien, se trata de que esas actividades de explicación y contrastación de hipótesis entran, de hecho, en la práctica científica, como componentes o momentos en una actividad más comprensiva de juicio o razonamiento práctico. Nos encontramos nuevamente con el pensamiento contextualista de Dewey que, en relación con la lógica, se expresa claramente en la siguiente cita: "la discusión filosófica sobre las distinciones y discusiones que aparece en las teorías lógicas depende de ubicarlas adecuadamente en su contexto temporal." Tal contexto temporal es, como vimos, la investigación, mientras que la negativa a realizar tal contextualización tiene, en su opinión, sus propios riesgos: "en ausencia de esa ubicación somos propicios a transferir los rasgos del asunto de una fase a los de otra, con un resultado confuso." (mw.10.320, ambas citas). Frente a la concepción retrospectiva y justificatoria presentada en el apartado anterior, resulta importante señalar la dimensión temporal aludida. En efecto, Dewey señala una y otra vez el hecho evidente, negado muchas veces por la filosofía, de que la investigación e incluso el razonamiento implica tiempo y por tanto una estructura temporal (cf. Volbers, 2018). Primero nos encontramos con el problema y sólo al final del proceso podemos sostener que algo es una solución.

En segundo lugar, la cita anterior parece contener una respuesta más profunda frente a las dudas escépticas acerca de la posibilidad del conocimiento. Si la ciencia es un modo de la práctica en el sentido explicitado, una actividad de toma de decisiones acerca de qué hacer, entonces el conocimiento científico es tan posible como cualquier otra forma de actividad inteligente en la que se toman decisiones fundadas. Dicho de otra manera, el hecho de que la deliberación práctica inteligente sea posible en los ámbitos en los que operan las investigaciones de sentido común sugiere que lo mismo ocurre con la ciencia, pues en ella nada extraordinario, nada esencialmente diferente sucede. En relación con ello, otro punto a destacar es el hecho de que dudar de o negar la existencia de tales investigaciones prácticas inteligentes equivale a sostener que la inteligencia no opera en ninguna cuestión práctica, sino que sólo contamos con el "impulso, capricho, hábito o convención.” Es interesante señalar que Dewey no rechaza la influencia del impulso, el capricho, el hábito ciego o la tradición. Es decir, no niega que a veces nuestras decisiones se apoyen más fuertemente en tales factores. Pero sostener que ello siempre es así parecería contradecir hechos evidentes. En su opinión, por ejemplo, es claro que resoluciones de problemas en disciplinas como la medicina se apoyan o tienden a apoyarse en investigaciones inteligentes más que en la mera tradición o el impulso.

Para avanzar en el análisis de la diferencia lógica entre tradición e impulso, por un lado, e inteligencia, por el otro, es posible apelar a la distinción que propone el propio Dewey entre una normatividad de facto y una normatividad de jure. Por normatividad nos referimos al hecho de que la conducta puede estar regulada 
por normas o principios. ${ }^{7}$ Tales normas pueden resultar vigentes tan solo como consecuencia del impulso, el capricho, el hábito irreflexivo o la tradición. En tal caso, la norma regula la acción pero sin derecho propio: lo hace de facto. La idea de Dewey es que sólo cuando tales principios son resultado de la investigación nos encontramos legítimamente con un principio que debe ser tenido en cuenta. Dicho de otro modo, y tal como ocurre con cualquier otra creencia, la única razón que tenemos para aceptar un principio es el hecho de que fuera resultado de la investigación y lo aceptamos porque la práctica ha mostrado que es el medio adecuado para cumplir con el fin propuesto por esa investigación, a saber, resolver satisfactoriamente el problema específico que la originó.

Esta apelación a la investigación inteligente práctica como elemento argumentativo a efectos de sostener la posibilidad del conocimiento aparece también al momento de dar cuenta de la posibilidad del conocimiento moral. En Teoría de la Valoración, y tras sostener que un juicio valorativo establece una norma, en el sentido de una condición a la que deben conformarse los actos futuros, Dewey sostiene lo siguiente:

Que las reglas son omnipresentes en todas las formas de relación humana es algo tan obvio que no necesita argumentación. No se restringen en absoluto a las actividades que cabe denominar 'morales'. Toda forma recurrente de actividad, en las artes y en las profesiones, desarrolla reglas que indican la mejor manera de cumplir los fines que se tienen a la vista. Tales reglas se usan como criterios o 'normas' para juzgar el valor de las distintas propuestas de conducta. No se puede negar que existen reglas para valorar los modos de conducta en diferentes campos como sabios o imprudentes, económicos o extravagantes, eficaces o fútiles. El problema no es su existencia como proposiciones generales (ya que toda regla de acción es general), sino el de si se limitan a reflejar costumbres, convenciones, tradiciones, o bien son capaces de establecer relaciones entre ciertas cosas tomadas como medios y otras entendidas como consecuencias, donde esas relaciones están fundadas ellas mismas en relaciones existenciales empíricamente verificadas y comprobadas, como normalmente se dice de las relaciones de causa y efecto (Dewey, 2008, 102. lw.13.209-210).

En esta cita volvemos a encontrarnos con la misma estrategia: el juicio moral es un juicio práctico y, por lo tanto, tiene notas en común con todos los juicios prácticos. Las normas existen, eso no puede negarse, pero la cuestión es si se limitan a establecer una normatividad de facto o si más bien pueden por derecho

\footnotetext{
${ }^{7}$ La palabra preferida por Dewey es principle, a la que contrasta con rule, en la medida en que comprende a esta última como una receta que estipula pasos fijos a seguir. En contraste con ello, un principio tiene una naturaleza metodológica o intelectual, es una herramienta intelectual para usar en la investigación moral (cf. lw.7. 279-278). Creemos que el término principio refleja efectivamente de mejor modo la posición de Dewey en comparación con el término norma, pero sin embargo lo conservamos por su vínculo con la idea de normatividad que se ha vuelto usual en la literatura filosófica.
} 
propio regir la conducta, es decir, si pueden ser el resultado de una investigación inteligente. Dewey no trata de deducir de alguna premisa la tesis de que las normas pueden regular legítimamente la conducta, sino que se remite a los hechos para mostrar la posibilidad de que ello ocurra. El tratamiento de las normas como instrumentos permite compararlos con cualquier herramienta que surge, se inventa en un contexto de actividad práctica y luego es mejorada con el desarrollo recurrente de actividades prácticas cuyos rasgos son suficientemente parecidos como para aplicar el mismo instrumento. Una vez que el instrumento ha sido creado y perfeccionado y que su valor ha sido recurrentemente probado en actividades subsecuentes, dicho instrumento se vuelve correcto, es decir, es el instrumento que hay que usar. Así, de acuerdo con Dewey, tanto un principio lógico como uno moral exhiben la misma normatividad razonable y siempre provisoria que cualquier instrumento: es razonable, deseable e incluso obligatorio usarlos porque son los medios adecuados para resolver los problemas que enfrentamos.

Nos encontramos aquí con una posición que resulta particularmente interesante cuando se refiere a los principios morales en sentido estricto. Lo primero que hay que tener en cuenta para valorar la concepción deweyana de los principios morales es, otra vez, su contexto de funcionamiento. Y su contexto no es el de un razonamiento en el sentido usual del término en el que el principio podría funcionar como premisa general desde la que luego, al aplicarla a un caso concreto (que se explicita en otras premisas), se deduce o se infiere una conclusión. Antes bien, su contexto es el de la deliberación práctica, entendida como un proceso mediante el cual se intenta incidir en un estado de cosas a partir de la decisión razonada acerca de qué hacer. En ese contexto, un principio funciona como una regla metodológica y no simplemente como una premisa. Para usar un concepto conocido, puede decirse que un principio es algo similar a un topos. Según van Eemeren, un topos puede considerarse como un movimiento argumentativo formulado como una regla de inferencia cuasi formal, que tiene dos funciones: una función de selección y una función de garantía (van Eemeren et al. 1996, cap. 2). En el contexto de un intercambio argumentativo o dialéctico, un topos es un principio material de inferencia -un hábito inferencial, podría decirse con Peirce- que sirve como guía para elegir las premisas que se necesitan para producir una conclusión, entre otras varias alternativas posibles, y ganar el debate. Esta es la función de selección, principalmente metodológica o heurística: es una guía, una ayuda en la selección de premisas. En el contexto de la lógica procesual de Dewey, estos topoi podrían entenderse como una herramienta para producir los datos que cooperarán en la resolución del problema. En otras palabras, el principio moral es un instrumento que coopera para abordar el problema en cuestión, al guiarnos en la búsqueda de los hechos del caso necesarios para abordar la cuestión. En efecto, refiriéndose a la "Regla de oro", Dewey afirma que

[...] nos da un punto de vista desde el cual considerar los actos; sugiere la necesidad de considerar cómo afectarían nuestros actos a los intereses de los demás tanto como a los nuestros; tiende a evitar la parcialidad en esa consideración y 
nos previene contra la inclinación a exagerar la importancia de una consecuencia particular de dolor o placer, simplemente porque sucede que nos afecta. En suma, la Regla de oro no dicta órdenes o mandatos especiales, sino aclara e ilumina las situaciones que requieren deliberación inteligente. (Dewey, 1965, 168. lw.7.280281)

La otra función del topoi, la función de garantía, se refiere al hecho de que en el contexto de una lógica dialéctica, un topos es también una premisa del argumento del cual se deduce la conclusión. Esta función no se niega en el contexto de la lógica de Dewey, pero se coloca en un papel secundario y reconstructivo. Esto es lo que se puede asumir al analizar las críticas de Dewey sobre la pretensión de identificar la lógica del silogismo práctico con la lógica de la deliberación. (cf. Dewey, 1950, 186-187. lw.12.166-167).

Por lo tanto, según Dewey, un principio es una herramienta, y como toda herramienta es el resultado de algún tipo de actividad práctica en curso, es un medio para lograr los fines de esa actividad. Más aún, se revalida como una buena herramienta. -y evoluciona- en otras aplicaciones. En sus palabras,

Una herramienta es algo para usar. Por lo tanto, también es algo que debe mejorarse observando cómo funciona. La necesidad de tal observación y mejora es indispensable si, como en el caso de los principios morales, la herramienta debe utilizarse en circunstancias no deseadas. La continuidad del crecimiento, no el atomismo, es, por lo tanto, la alternativa a la fijación de principios y objetivos. (lw.14.168)

De este modo, según Dewey, para que la deliberación sea correcta no necesita principios externos fijos sino principios en evolución con los que analizar cada nuevo problema. Esto implica que los principios siempre están abiertos a revisión $y$, por consiguiente, son siempre hipotéticos, al igual que las generalizaciones empíricas en el contexto de las investigaciones científicas.

Otro punto interesante es que, en este marco, los principios morales no se consideran como establecidos previamente, como algo ya hecho y fijo, sino como algo que cambia y evoluciona, de acuerdo con su naturaleza instrumental: los nuevos problemas pueden requerir nuevos o mejores instrumentos, y de este modo se introduce la idea misma de novedad y creatividad en un campo tradicionalmente considerado como ajeno a ella como es el campo de la moral. Esta necesidad de creatividad en el desarrollo de principios, valores o fines no es un fin en sí mismo como, por ejemplo, suele ser en el caso de las prácticas artísticas. Es una consecuencia de la comprensión de la deliberación moral no como una deducción de una acción buena o correcta a partir de un principio, sino como el intento de resolver algún problema. Mientras surjan nuevos problemas, la necesidad de nuevos medios o instrumentos para resolverlos seguirá estando vigente. En efecto, como recuerda Dewey, 
[s]i el hábito falla, la única alternativa para no obrar por capricho y azar es la reflexión. Y la reflexión acerca de lo que debe hacerse es idéntica a la formación de fines. Más aún, cuando el cambio social es grande y sugiere una gran variedad de objetivos contradictorios, la reflexión no puede limitarse a escoger uno de ellos de entre el conjunto que le sugieren las condiciones. El pensamiento tiene que operar creativamente para formar nuevos fines. (Dewey, 1965, 50. lw.7.185)

Para finalizar, queremos destacar dos aspectos de la concepción deweyana de la deliberación que creemos valiosos. Se trata, en primer lugar, de la idea de que la deliberación es tanto sobre medios como sobre fines. Más aún, en opinión de Dewey los fines se evalúan en las mismas valoraciones en las que se consideran los medios. En este sentido, un fin, lo mismo que un principio o un valor, no puede considerarse como una premisa en el razonamiento práctico como suele hacerse en la literatura actual (cfr. Macagno y Walton, 2018; Walton, 1990; Walton, 2007), sino como algo que se establece en el mismo proceso. En segundo lugar, en la medida en que la deliberación es una respuesta a una situación problemática en la que hay que hacer algo, pues algo ocurrirá de todos modos y se busca producir o evitar un cierto resultado, una reconstrucción de la estructura de la deliberación debería tener en cuenta el punto de partida no siempre explícito de que algo puede y debe hacerse. En efecto, esa es la nota distintiva de los juicios prácticos. De este modo, las expresiones normativas no son algo que mágicamente aparece en el resultado o la conclusión de una deliberación práctica, sino algo que caracteriza desde el comienzo a la deliberación pues ésta siempre se desarrolla por referencia a la situación inicial. Es decir, no hay ningún salto lógico entre ser y deber ser, sino que se trata siempre de deliberar sobre la base de que algo hay que hacer, de que algo puede y tal vez debe hacerse.

\section{Conclusiones}

De acuerdo con lo señalado en las páginas precedentes, en el marco de la filosofía de Dewey el asunto de la normatividad tiene un espesor doble. Por un lado ha sido uno de los aspectos más cuestionados de su posición teórica, al punto que le han llegado a señalar que no ofrece una solución adecuada al problema de la fundamentación de los principios morales. Por otro lado, considerando junto con Dewey que la instrumentalidad de la ciencia es el problema más acuciante de la vida contemporánea, es un tema de ineludible importancia política y moral. En este sentido, abordar la pregunta por el carácter vinculante, legítimo y racional de las normas ha sido y sigue siendo una tarea urgente.

Bajo estas consideraciones, el objetivo principal del artículo ha sido reconstruir la concepción deweyana de normatividad. El recorrido propuesto nos ha permitido sostener dos afirmaciones centrales, a saber: (i) que la normatividad debe comprenderse en términos eminentemente prácticos, comunes a la investigación científica y a la valoración moral y política; y (ii) que el fundamento del 
carácter normativo, vinculante y legítimo de los principios (lógicos o morales, dado que comparten una misma pauta general) es la propia investigación. Dicho en otros términos, hemos planteado que según Dewey la normatividad está enraizada en la experiencia, pues su material proviene sólo de allí, y que se funda en la investigación acumulada y empíricamente regulativa. En este sentido, la investigación se instituye como la sola alternativa a la acción guiada por el mero impulso, el capricho, el hábito ciego y/o las tradiciones. Este punto resulta importante pues sugiere la naturaleza política de la propuesta deweyana. No es que siempre los seres humanos decidamos sobre los principios que guían nuestras acciones a través de la deliberación y la investigación. Lo que Dewey sugiere es que es posible y, más aún, deseable hacerlo de este modo en la medida en que nos comprometemos con la tarea de hacer del mundo un lugar mejor, un lugar en el que podamos y queramos vivir juntos. Es este proyecto moral y político, que apela a la inteligencia y a la ciencia como herramientas para abordar los conflictos humanos, el que se expresa en la siguiente cita que reproducimos para terminar:

[...] cada generación, especialmente una que viva en una época como la presente, tiene la responsabilidad de reajustar su heredado caudal de principios morales y de reconsiderarlos en relación con las condiciones y necesidades contemporáneas. Sería estúpido suponer que esto significa que todos los principios morales están tan relacionados con un determinado estado de sociedad que no tienen fuerza obligatoria en ninguna condición social. La obligación es descubrir qué principios son pertinentes a nuestro propio tiempo social. [...] El reconocimiento de su íntima y vital relación con las fuerzas sociales creará e intensificará la búsqueda de los principios que son verdaderamente pertinentes en nuestros días. (Dewey, 1965, 172. lw.7.238).

\section{Referencias bibliográficas}

Colapietro, Vincent (2018). The Actuality of Philosophy Thought Over Once Again. The Journal of Speculative Philosophy, 32(1), 3-20.

Dewey, John. The Collected Works of John Dewey, 1882-1953 (1967-1987). The Early Works, 1882-1898, 1967-1972 (5 vols.); The Middle Works, 1899-1924, 19761983 (15 vols.), The Later Works, 1925-1953, 1981-1991 (17 vols.). Carbondale: Southern Illinois University Press.

Dewey, John (1950). Lógica. Teoría de la Investigación [1938]. Prólogo y traducción de Eugenio Imaz. México: FCE.

Dewey, John (1965). Teoría de la vida moral [1932]. Traducción de Rafael Castillo Dibildox. México D.F.: Herrero Hermanos.

Dewey, John (2008). Teoría de la valoración. Un debate con el positivismo sobre la dicotomía de hechos y valores. [1939]. Madrid: Biblioteca Nueva. 
Dewey, John (2012). Unmodern philosophy and modern philosophy. Carbondale: Southern Illinois University Press.

di Berardino, A. y Faerna, Á. M. (2008). Introducción a Dewey. En John Dewey, Teoría de la valoración. Un debate con el positivismo sobre la dicotomía de hechos y valores. [1939]. Madrid: Biblioteca Nueva.

Eemeren van, Fran; Grootendorst, Rob, Snoeck Henkemans, Francisca et al (1996). Fundamentals of Argumentation Theory. A Handbook of Historical Backgrounds and Contemporary Developments. Mahwah, NJ: Erlbaum.

Faerna, Á. M. (2006). Significado y valor: La crítica pragmatista al emotivismo. Quaderns de filosofia i ciència, 36, 27-39.

Faerna, Á. M. (2011). Dewey's Value Theory and the Analytic Tradition of Moral Philosophy. En L. A. Hickman, K. P. Skowronski, J. A. Rea (Eds.), The Continuing Relevance of John Dewey: Reflections on Aesthetics, Morality, Science, and Society (p. 13). Amsterdam, New York: Rodopi Press.

Knorr Cetina, Karin (2005). La fabricación del conocimiento. Un ensayo sobre el carácter constructivista y contextual de la ciencia [1981]. Bernal: Universidad Nacional de Quilmes.

Kusch, Martin (1995). Psychologism: A Case Study in the Sociology of Philosophical Knowledge. Londres: Routledge.

Latour, Bruno, Woolgar, Steve (1995). La Vida en el Laboratorio: la Construcción de los Hechos Científicos [1986]. Madrid. Alianza Editorial.

Laudan, Larry (1980). Why was the logic of discovery abandoned? En Thomas Nickles (Ed.), Scientific Discovery, Logic and Rationality (pp. 173-184). Dordrecht/Boston/London: Reidel.

Lewis, Clarence Irwing (1929). Review of John Dewey's The Quest for Certainty. En Collected Papers of Clarence Irving Lewis (1970). Stanford, Calif.: Stanford University Press.

Macagno, Fabrizio, Walton, Douglas (2018). Practical Reasoning Arguments: A Modular Approach. Argumentation, 32(4), 519-547.

Marcuse, Herbert (1941). Review: Dewey, John, Theory of Valuation. Zeitschrift für Sozialforschung, 9(1), 144-148.

Naishtat, Francisco (2006). Problemas filosóficos en la acción individual y colectiva. Buenos Aires: Prometeo, 2006.

Peirce, Charles Sanders (1965). Collected Papers of Charles Sanders Peirce. Cambridge, Mass: Belknap Press of Harvard University Press.

Reichenbach, Hans (1938). Experience and Prediction. Chicago: University of Chicago Press.

Salmon, Wesley (1973). Logic. Englewood Cliffs: Prentice-Hall. 
Schickore, Jutta, Steinle, Friedrich (Eds.) (2006) Revisiting Discovery and Justification: Historical and Philosophical Perspectives on the Context Distinction. Springer.

Sinclair, Robert (2014). Dewey and White on Value, Obligation, and Practical Judgment. SATS, 15(1), 39-54.

Stevenson, Charles (1944). Ethics and Language. New Haven: Yale University Press.

Toulmin, Stephen E. (2003). The Uses of Argument. Cambridge. Cambridge University Press. doi:10.1017/CBO9780511840005

Volbers, Jörg (2018). Reclaiming the Power of Thought. European Journal of Pragmatism and American Philosophy [Online], X-2. URL: http://journals.openedition.org/ ejpap/1327

Walton, Douglas (2007). Evaluating Practical Reasoning. Synthese, 157(2),197-240.

Walton, Douglas (1990). Practical Reasoning: Goal-Driven, Knowledge-Based, Action-Guiding Argumentation. Ethics, 102(1), 179-180.

White, Morton (1949). Value and obligation in Dewey and Lewis. The Philosophical Review, 58(4), 321-329. 